\title{
Imaging Diagnosis of Emphysematous Pyelonephritis in a Non-Diabetic Dog
}

\author{
Heesu Kim*, Kija Lee**, Jinyoung Chung*, Jinok Ahn*, Inchul Park* and Sooyoung Choi*1 \\ *College of Veterinary Medicine, Kangwon National University, Chuncheon 24341, Korea \\ **College of Veterinary Medicine, Kyungpook National University, Daegu 41566, Korea
}

(Received: July 08, 2019 / Revised: July 06, 2020 / Accepted: August 18, 2020)

\begin{abstract}
An 11-year-old, $4.3 \mathrm{~kg}$, mixed breed, intact female dog exhibiting vomiting and a reduced appetite was presented. On physical examination, palpation of the right kidney elicited discomfort. Blood analysis revealed leukocytosis with neutrophilia, and elevation of alkaline phosphatase and blood urea nitrogen. Escherichia coli was detected in urine culture, whereas urine analysis showed absence of glucose. Radiography and ultrasonography suggested that there was gas in the right renal parenchyma, and computed tomography was performed while the dog was awake to investigate the suspected gas in the right kidney. After unilateral ureteronephrectomy, a histological diagnosis of right emphysematous pyelonephritis (EPN) was made. EPN refers to a severe necrotizing infection of the renal parenchyma with gas accumulation in the tissue. It is rare in both human and veterinary medicine, and almost all EPN patients have uncontrolled diabetes mellitus. This case report describes the imaging-based diagnosis of a rare occurrence of unilateral EPN in a non-diabetic dog.
\end{abstract}

Key words : canine, computed tomography, emphysematous pyelonephritis, non-diabetic dog, radiography.

\section{Introduction}

Emphysematous pyelonephritis (EPN) is a severe necrotizing infection of the renal parenchyma involving gas accumulation in the renal parenchyma, collecting system, or perinephric tissue (7). In human medicine, women are more likely to be affected by EPN, but no sex predisposition has been reported in the veterinary literature $(12,17)$. Clinical signs are comparable to those of other types of pyelonephritis, including fever, pyuria, and flank pain (5). Reported mortality rates have decreased since the $1970 \mathrm{~s}$, and are currently approximately $13.5 \%$ in humans (14). Ureteronephrectomy and percutaneous drainage have been the recommended treatments (4).

In both human and veterinary medicine, most EPN patients have uncontrolled diabetes mellitus (DM) as an underlying disease, and EPN is evidently rare in patients without DM $(1,3,8)$. While DM is the most commonly associated comorbidity, the pathogenesis of EPN is not fully understood. Severe necrotizing infection and impaired vascular supply are characteristic features of EPN (7). Impaired tissue and vascular responses enable organisms capable of producing carbon dioxide to use the necrotic tissue as a substrate for gas generation. Local factors (diabetic glomerulopathy due to high tissue glucose level, urinary obstruction) and systemic factors (infection with gas-forming bacteria such as Escherichia coli, immunosuppressive diseases) lead to tissue and vascular damage $(3,16)$. An impaired host response is a reasonable explanation for EPN even in patients without DM or

${ }^{1}$ Corresponding author.

E-mail : choisooyoung@kangwon.ac.kr evidence of infection (16).

In previous veterinary reports, EPN was diagnosed via radiological depictions of gas superimposed over both kidneys, multiple round to linear gas opacities within mildly enlarged renal silhouettes, and/or multifocal gas lucencies in the region of the kidney $(3,6,8)$. On ultrasonography, multiple hyperechoic foci with reverberation artifact in the renal cortices, and gas at the corticomedullary junction with severe hydronephrosis have been reported $(3,6,8)$. Imaging modalities such as radiography, ultrasonography, and computed tomography (CT) are essential for the diagnosis of EPN (19). EPN can be diagnosed when an abnormal radiolucent area in renal or perinephric tissue is detected via plain radiography and/or ultrasonography. Other organs normally containing gas such as the intestine can be superimposed over the kidney on radiography. On ultrasonography, intestinal gas can cause acoustic shadow and reverberation artifacts. Notably, however, in a systematic review, abdominal radiography findings were found to be accurate in only $69 \%$ of cases, and ultrasound findings in $65 \%$ (14). In contrast, the sensitivity and specificity of CT with regard to identifying gas patterns is reportedly almost $100 \%(1,4)$. Accordingly, CT has been recommended as the imaging modality of choice for the early diagnosis of EPN $(6,18)$.

Herein we describe the imaging diagnosis of a case of EPN in a non-diabetic dog via radiography, ultrasonography, and CT performed while the dog was awake.

\section{Case Report}

An 11-year-old, $4.3 \mathrm{~kg}$, mixed breed, intact female dog was presented exhibiting vomiting, diarrhea, and anorexia. Abnormalities detected via physical examination included 


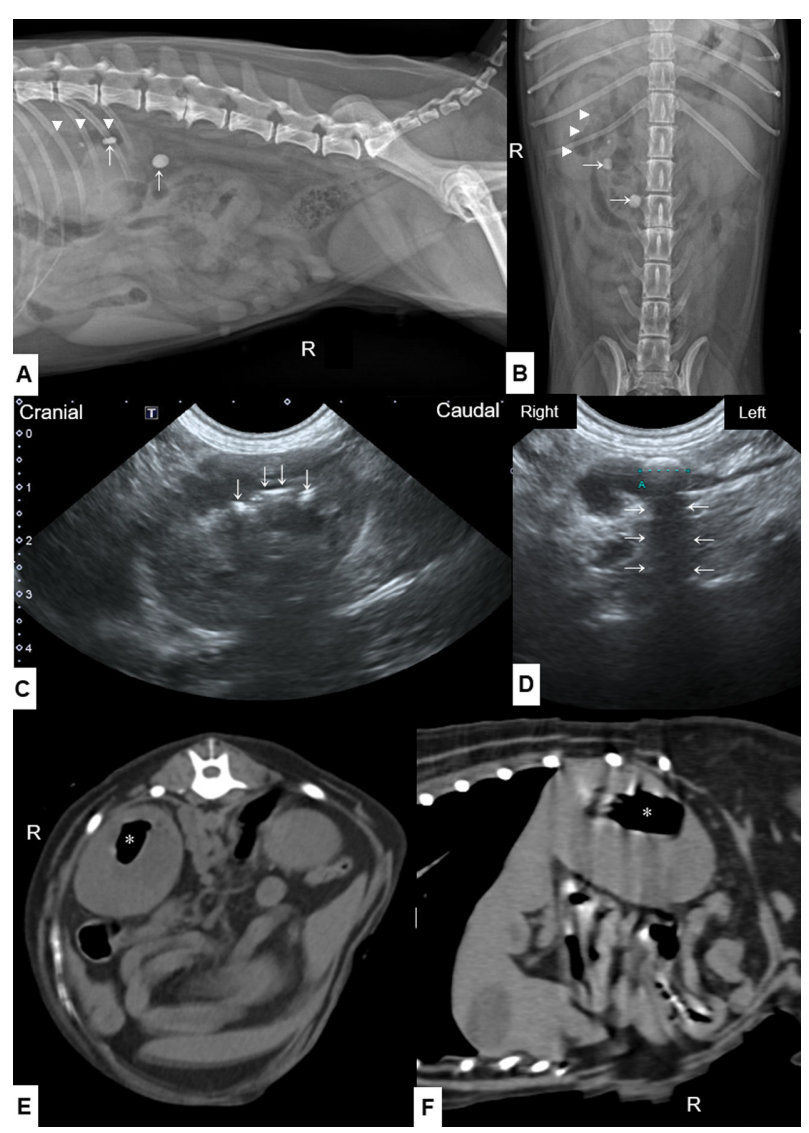

Fig 1. A, B. Right lateral and ventrodorsal abdominal radiographs. Abdominal radiographs shows free gas (arrows) within the right kidney and calculi (arrowheads) in the right ureter. The kidneys are of normal size and shape. C. Sagittal ultrasonographic image of the right kidney. Multiple reverberation artifacts are present (arrows). D. Sagittal ultrasonographic image of the right urethra. Proximal urethral calculi of approximately 0.7 $\mathrm{cm}$ are seen, in addition to acoustic shadowing (arrows). E, F. Transverse and sagittal computed tomography images. Computed tomography reveals the accumulation of gas within the parenchyma of the right kidney (asterisk).

$7 \%$ dehydration, and pain on palpation of the right kidney. A complete blood count revealed leukocytosis $\left(20.8 \times 10^{3} / \mu \mathrm{L}\right.$, reference interval $\left.[\mathrm{RI}] 5.0-16.7 \times 10^{3} / \mu \mathrm{L}\right)$ with neutrophilia $\left(13.2 \times 10^{3} / \mu \mathrm{L}\right.$, RI $\left.2.9-11.6 \times 10^{3} / \mu \mathrm{L}\right)$, and lymphocytosis $\left(6.53 \times 10^{3} / \mu \mathrm{L}\right.$, RI $\left.1-5 \times 10^{3} / \mu \mathrm{L}\right)$ and thrombocytopenia $(86 \times$ $10^{3} / \mu \mathrm{L}$, RI $\left.148-484 \times 10^{3} / \mu \mathrm{L}\right)$ were detected. The serum biochemical profile included elevated alkaline phosphatase (749 U/L, RI 23-212 U/L), blood urea nitrogen (48 mg, RI 7--27 $\mathrm{mg}$ ), and phosphorus (11.1 mg, RI 2.5-6.8 mg).

Right lateral and ventrodorsal abdominal radiographs depicted multifocal gas lucencies in the region of the right kidney, and calculi in the right ureter and right renal parenchyma (Fig 1A and 1B). The gas lucencies needed to be differentiated from focal intestinal dilation, and abdominal ultrasonography revealed a smooth and hyperechoic interface with mild reverberation artifact on the right kidney (Fig 1C). Calculi were observed in the right proximal ureter (Fig 1D). The bladder wall was slightly thickened and a focal gascontaining dilation of the small intestine was not observed. To confirm the suspected gas indicated by radiography and

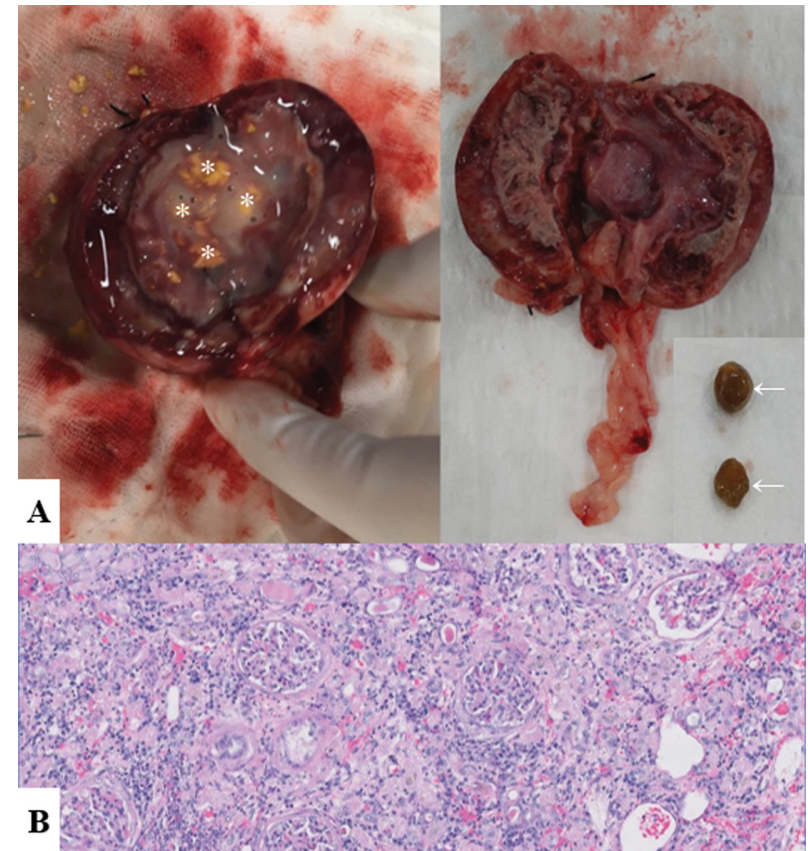

Fig 2. A. Right kidney and ureter. Pathological examination of the kidney reveals features of abscess formation (asterisk) and two calculi in the ureter (arrow). B. Cortex of the right kidney. As inflammation extends into the cortex, it effaces the interstitium multifocally. Inflammatory infiltrates predominantly consist of neutrophils mixed with foamy macrophages, numerous components associated with hemorrhage, and cellular debris. There are numerous reactive fibroblasts. Hematoxylin and eosin stain, bar $=50 \mu \mathrm{m}$.

ultrasonography, pre-contrast CT using a 16-channel multidetector (Alexion, Toshiba; Otawara, Japan) was performed while the dog was awake (without anesthesia or sedation). Gas and micro-calculi were identified in renal parenchyma with surrounding fatty stranding, and renal pelvic dilation and proximal ureter dilation due to two ureteral calculi were apparent.

Urine was sampled under ultrasound guidance, and urinary analysis revealed a specific gravity of 1.023, hemoglobinuria, urobilinogen, proteinuria, and leukocytes. No red blood cells or glucose were observed, but rod-shaped bacteria were detected. Urine culture yielded $E$. coli that were susceptible to enrofloxacin and cephalothin.

EPN was suspected based on the above imaging findings and urinalysis, and unilateral ureteronephrectomy was performed under general anesthesia. Gross examination of the removed kidney revealed abscess formation and two calculi in the ureter (Fig 2A). Histological examinations showed that the architecture of the kidney was extensively distorted by a marked, coalescing inflammatory infiltrate (Fig 2B). The infiltrate was most severe in the renal pelvis, and inflammation extended into the cortex. A bacterial etiology was strongly suspected. There was severe pyelonephritis and inflammation in the ureter, with focal ulceration.

The dog was hospitalized for the subsequent 4 days, during which it was treated with the antibiotic enrofloxacin. At a follow-up examination 2 weeks postoperatively, there were no clinical symptoms. At the 1-year follow-up, no specific 
changes were observed.

\section{Discussion}

In humans, diagnostic imaging tools must be used to confirm the presence of gas in the renal parenchyma, because the clinical signs and laboratory findings associated with the condition are similar to those associated with renal sepsis (19). A three-stage classification system has been applied in humans based on plain radiographic characteristics. Stage I is characterized by gas in the renal parenchyma or perinephric tissue, stage II by gas in the kidney and its surroundings, and stage III by extension of gas through the fascia or bilateral disease. However, it can be difficult to distinguish a necrotic gas-filled area from gas in the bowel or gas from acoustic shadowing due to calculi, via radiography (7). The dog in the present case was considered to be stage I, but it was necessary to distinguish its condition from intestinal gas accumulation.

In 1996, Wan et al. described two types of EPN based on CT parameters (20). Type I is characterized by renal parenchymal destruction with gas but no fluid, and type II by renal parenchymal or peri-renal parenchymal destruction with gas and fluid. In 2000, Huang et al. (4). described a series of classes of EPN based on CT findings. Class 1 is characterized by gas in the collecting system only, class 2 by gas in the renal parenchyma only, class $3 \mathrm{~A}$ by extension of gas or abscess to the perinephric space, class $3 \mathrm{~B}$ by extension of gas or abscess to the para-renal space, and class 4 by bilateral EPN or solitary kidney with EPN. Such classification systems assist in the determination of treatment strategies $(4,19)$.

In the present case, we had to perform CT for the diagnosis of EPN and determination of the optimal treatment strategy. Gas was identified in the renal parenchyma, and the dog's condition was classified as class 2 according to the classification system described by Huang et al. (4). A tendency toward increasing mortality from class 1 to class 4 EPN has been reported (4). In humans, mortality rates of approximately $10 \%$ have been suggested, and systolic blood pressure $<90 \mathrm{mmHg}$, unconsciousness, high creatinine level, thrombocytopenia, bilaterality, and medical treatment alone are evidently associated with a higher risk of mortality $(2,14)$. Mortality rates and prognostic factors in veterinary patients are unknown. The dog in the present case was class 2 and only exhibited one risk factor (thrombocytopenia).

Due to the risks associated with anesthesia, CT was performed while the dog was awake. General anesthesia is usually essential in small animals undergoing CT, but it can be risky in substantially compromised patients (10). There have been reports on successful CT in awake dogs and cats for the evaluation of upper airway disease, intrathoracic disease, and acute abdominal symptoms $(9,10,11,13,15)$. The present case suggests that CT can be performed in the awake state in dogs for the diagnosis of EPN.

\section{Conclusion}

Unilateral EPN rarely occurs in dogs without DM. In dogs, CT evaluation in the awake state can be used in conjunction with radiography and ultrasonography to obtain a more detailed diagnosis, determine an appropriate choice of treatment, and predict the prognosis.

\section{Acknowledgement}

This study was supported by 2018 Research Grant from Kangwon National University (520180047).

\section{References}

1. Dhabalia JV, Nelivigi GG, Kumar V, Gokhale A, Punia MS, Pujari N. Emphysematous pyelonephritis: tertiary care center experience in management and review of the literature. Urol Int 2010; 85: 304-308.

2. Falagas ME, Alexiou VG, Giannopoulou KP, Siempos II. Risk factors for mortality in patients with emphysematous pyelonephritis: a meta-analysis. J Urol 2007; 178: 880-885.

3. Gould EN, Cohen TA, Trivedi SR, Kim JY. Emphysematous pyelonephritis in a domestic shorthair cat. J Feline Med Surg 2016; 18: 357-363.

4. Huang JJ, Tseng CC. Emphysematous pyelonephritis: clinicoradiological classification, management, prognosis, and pathogenesis. Arch Intern Med 2000; 160: 797-805.

5. Khaira A, Gupta A, Rana DS, Gupta A, Bhalla A, Khullar D. Retrospective analysis of clinical profile prognostic factors and outcomes of 19 patients of emphysematous pyelonephritis. Int Urol Nephrol 2009; 41: 956-966.

6. Lim JS, Yoon Y, Jung D, Yeon S, Lee H. Emphysematous pyonephrosis associated with extrahepatic portosystemic shunt in a dog. J Vet Med Sci 2016; 78: 697-700.

7. Michaeli J, Mogle P, Perlberg S, Heiman S, Caine M. Emphysematous pyelonephritis. J Urol 1984; 131: 203-208.

8. Moon R, Biller DS, Smee NM. Emphysematous cystitis and pyelonephritis in a nondiabetic dog and a diabetic cat. J Am Anim Hosp Assoc 2014; 50: 124-129.

9. Ngwenyama TR, Herring JM, O’Brien M, Hartman SK, Galloway KA, O'Brien RT. Contrast-enhanced multidetector computed tomography to diagnose pulmonary thromboembolism in an awake dog with pyothorax. J Vet Emerg Crit Care 2014; 24: 731-738.

10. Oliveira CR, Mitchell MA, O’Brien RT. Thoracic computed tomography in feline patients without use of chemical restraint. Vet Radiol Ultrasound 2011; 52: 368-376.

11. Oliveira CR, Ranallo FN, Pijanowski GJ, Mitchell MA, O'Brien MA, McMichael M, Hartman SK, Matheson JS, O'Brien RT. The VetMousetrap: a device for computed tomographic imaging of the thorax of awake cats. Vet Radiol Ultrasound 2011; 52: 41-52.

12. Root CR, Scott RC. Emphysematous cystitis and other radiographic manifestations of diabetes mellitus in dogs and cats. J Am Vet Med Assoc 1971; 158: 721-728.

13. Shanaman MM, Hartman SK, O’Brien RT. Feasibility for using dual-phase contrast-enhanced multi-detector helical computed tomography to evaluate awake and sedated dogs with acute abdominal signs. Vet Radiol Ultrasound 2012; 53: 605-612.

14. Somani BK, Nabi G, Thorpe P, Hussey J, Cook J, N'Dow J. Is percutaneous drainage the new gold standard in the management of emphysematous pyelonephritis? Evidence from a systematic review. J Urol 2008; 179: 1844-1849.

15. Stadler K, O'Brien R. Computed tomography of nonanesthetized cats with upper airway obstruction. Vet Radiol Ultrasound 2013; 54: 231-236. 
16. Tang HJ, Li CM, Yen MY, Chen YS, Wann SR, Lin HH, Lee SS, Liu YC. Clinical characteristics of emphysematous pyelonephritis. J Microbiol Immunol Infect 2001; 34: 125130 .

17. Thomas AA, Lane BR, Thomas AZ, Remer EM, Campbell SC, Shoskes DA. Emphysematous cystitis: a review of 135 cases. BJU Int 2007; 100: 17-20.

18. Tsu JH, Chan CK, Chu RW, Law IC, Kong CK, Liu PL,
Cheung FK, Yiu MK. Emphysematous pyelonephritis: an 8year retrospective review across four acute hospitals. Asian J Surg 2013; 36: 121-125.

19. Ubee SS, McGlynn L, Fordham M. Emphysematous pyelonephritis. BJU Int 2011; 107: 1474-1478.

20. Wan YL, Lee TY, Bullard MJ, Tsai CC. Acute gas-producing bacterial renal infection: correlation between imaging findings and clinical outcome. Radiology 1996; 198: 433-438. 